\title{
Membrane-bound and soluble Fas ligands have opposite functions in photoreceptor cell death following separation from the retinal pigment epithelium
}

\author{
H Matsumoto ${ }^{1}$, Y Murakami ${ }^{1}$, K Kataoka ${ }^{1}$, S Notomi ${ }^{1}$, D Mantopoulos ${ }^{1}$, G Trichonas ${ }^{1}$, JW Miller ${ }^{1}$, MS Gregory ${ }^{2}$, BR Ksander ${ }^{2}$, \\ A Marshak-Rothstein ${ }^{3}$ and DG Vavvas ${ }^{*, 1}$
}

Fas ligand (FasL) triggers apoptosis of Fas-positive cells, and previous reports described FasL-induced cell death of Fas-positive photoreceptors following a retinal detachment. However, as FasL exists in membrane-bound (mFasL) and soluble (sFasL) forms, and is expressed on resident microglia and infiltrating monocyte/macrophages, the current study examined the relative contribution of $\mathrm{mFasL}$ and $\mathrm{sFasL}$ to photoreceptor cell death after induction of experimental retinal detachment in wild-type, knockout (FasL - / ) , and mFasL-only knock-in ( $\Delta \mathrm{CS}$ ) mice. Retinal detachment in FasL - /- mice resulted in a significant reduction of photoreceptor cell death. In contrast, $\Delta$ CS mice displayed significantly more apoptotic photoreceptor cell death. Photoreceptor loss in $\Delta \mathrm{CS}$ mice was inhibited by a subretinal injection of recombinant sFasL. Thus, Fas/FasLtriggered cell death accounts for a significant amount of photoreceptor cell loss following the retinal detachment. The function of Fas $L$ was dependent upon the form of Fas $L$ expressed: $m F a s L$ triggered photoreceptor cell death, whereas $s F a s L$ protected the retina, indicating that enzyme-mediated cleavage of FasL determines, in part, the extent of vision loss following the retinal detachment. Moreover, it also indicates that treatment with SFasL could significantly reduce photoreceptor cell loss in patients with retinal detachment.

Cell Death and Disease (2015) 6, e1986; doi:10.1038/cddis.2015.334; published online 19 November 2015

Separation of photoreceptors from underlying retinal pigment epithelium (RPE), as seen rhegmatogenous retinal detachment, ${ }^{1}$ causes photoreceptor cell death, resulting in permanent vision loss. In a majority of cases, photoreceptor cell death occurs even if the retina is successfully reattached surgically. Separation of photoreceptors from the RPE also contributes to photoreceptor cell death in age-related macular degeneration, ${ }^{2}$ diabetic retinopathy, ${ }^{3}$ and retinopathy of prematurity. ${ }^{4}$ Therefore, it is important to define the mechanism(s) of photoreceptor cell death in the detached retina and establish therapeutic targets that prevent photoreceptor loss and the subsequent decrease in visual acuity.

Fas ligand (FasL) exists as a trimer in the cell membrane, whereas the Fas receptor (FasR or Fas) is expressed as a monomer. When FasL-positive cells come in contact with Fas-positive cells, Fas/FasL binding causes trimerization of Fas receptors that signals the binding of Fas-associated death domain (FADD) adaptor proteins; this triggers a sequential signaling cascade that recruits and activates caspase 8 , caspase 3 , and finally caspaseactivated DNAse (CAD) that ultimately enters the nucleus and cleaves DNA, resulting in apoptotic cell death. ${ }^{5-7}$ Although Fas signaling is mainly associated with this apoptotic cell death pathway, it has also been reported that, when FasL triggers Fas receptors in cells that inhibit or lack caspase 8 , an alternative death pathway is activated that is mediated by receptor interacting protein (RIP) kinase, leading to necrotic cell death. ${ }^{8-11}$ Thus, Fas signaling can induce not only apoptosis but also necrosis. It is important to understand whether photoreceptors die via apoptosis or necrosis following the retinal detachment, as necrotic cell death typically causes infiltration of inflammatory cells that may cause bystander death of surrounding normal cells, increasing loss of photoreceptors.

FasL is a type II transmembrane protein in the TNF family, and like many genes in this group, FasL exists in several different forms. ${ }^{12}$ The membrane-bound form (mFasL) can be cleaved from the cell surface by metalloproteinases to produce a truncated soluble product (sFasL) derived from the extracellular domain. ${ }^{13}$ Prior studies demonstrated that apoptosis triggered by FasL requires extensive oligomerization of the Fas receptor to activate the death-inducing signaling complex (DISC). ${ }^{14}$ Although both $\mathrm{mFasL}$ and sFasL contain

\footnotetext{
${ }^{1}$ Angiogenesis Laboratory, Department of Ophthalmology, Massachusetts Eye and Ear Infirmary, Harvard Medical School, Boston, MA, USA; ${ }^{2}$ Department of Ophthalmology, Massachusetts Eye and Ear Infirmary, Harvard Medical School, Schepens Eye Research Institute, Boston, MA, USA and ${ }^{3}$ Department of Medicine, University of Massachusetts Medical School, Worcester, MA, USA

*Corresponding author: DG Vavvas, Department of Ophthalmology, Harvard Medical School, 325 Cambridge Street, Boston, MA 02114 , USA. Tel: 617 573 6874; Fax: 617573 3011; E-mail: vavvas@meei.harvard.edu

Abbreviations: FasL, Fas ligand; mFasL, membrane-bound FasL; sFasL, soluble FasL; RPE, retinal pigment epithelium; FADD, Fas-associated death domain; CAD, caspase-activated DNAse; RIP, receptor interacting protein; DISC, death-inducing signaling complex; PBS, phosphate-buffered saline; TUNEL, TdT-dUTP terminal nickend labeling; ONL, outer nuclear layer; INL, inner nuclear layer; TEM, transmission electron microscopy; MCP-1, monocyte chemoattractant protein 1; IL-6, interleukin 6; LCM, laser capture microdissection; qPCR, quantitative real-time PCR; WT, wild type; CTR, control; siFAS, small interfering RNA against the Fas-receptor transcript Received 29.7.15; revised 09.10.15; accepted 12.10.15; Edited by RA Knight
} 
the trimerization domain and can bind the Fas receptor, the naturally cleaved form of SFasL is unable to oligomerize the Fas receptor and trigger apoptosis. ${ }^{14-16}$ For this reason, $\mathrm{mFasL}$ but not sFasL induces apoptosis in Fas-positive cells. In addition, reports indicate that sFasL blocks mFasLmediated apoptosis via steric hindrance when sFasL binds the Fas receptor and physically blocks the binding of $\mathrm{mFas}$ and oligomerization of the Fas receptor. ${ }^{17,18}$

Within the eye, Fas is expressed widely on cells in the anterior and posterior segment, whereas FasL has very limited expression and is found only on corneal epithelial cells, microglia, astrocytes, and RPE cells. The constitutive expression of FasL on corneal epithelial cells and RPE cells is necessary to maintain ocular immune privilege by inducing apoptosis of infiltrating Fas-positive inflammatory cells, which limits inflammation and subsequent tissue damage of ocular tissues. ${ }^{19}$ Although FasL limits inflammation, other reports indicate that $\mathrm{mFasL}$ promotes inflammation, and that $s F a s L$ is non-inflammatory or blocks mFasL-triggered inflammation. Therefore, the overall function of FasL is the result of the separate contributions of $\mathrm{mFasL}$ and sFasL, which have opposing functions in apoptosis and inflammation. ${ }^{17,18}$

The function of Fas/FasL in photoreceptor death was examined by our group in a rat model of retinal detachment, ${ }^{5}$ as well as other groups who observed a significant decrease in photoreceptor apoptosis in FasL ${ }^{\text {gld }}$ and Fas ${ }^{I p r}$ mutant mice. ${ }^{6,20}$ However, although these data demonstrate clearly that this pathway contributes to photoreceptor cell loss in detached retinas, these studies did not examine the contribution of the different forms of FasL (mFasL and sFasL). Moreover, these previous studies used FasL ${ }^{\text {gld }}$ and Fas ${ }^{l p r}$ mutant mice, which have specific point mutations in FasL and Fas (gld and Ipr mutations, respectively) that reduce but do not block completely Fas/FasL signaling; ${ }^{21}$ thus, the overall contribution of FasL in photoreceptor cell death is not completely known.

In our current study, we examined the overall contribution of FasL using FasL-knockout (FasL - / - ) mice and the relative contribution of $\mathrm{mFasL}$ and $\mathrm{sFasL}$ in the death of photoreceptors following experimentally induced retinal detachment. Fas/FasL signaling was completely eliminated in FasL - /mice and SFasL was eliminated in $\triangle \mathrm{CS}$ mice that possess an exchange knock-in mutation in the FasL metalloproteinase cleavage site, producing mice that express increased levels of mFasL and no sFasL. ${ }^{22}$ The potential neuroprotective effects of sFasL in photoreceptor cell death were also examined during retinal detachment.

\section{Results}

FasL mediates photoreceptor cell death induced by retinal detachment. To determine the relative contribution of FasL, mFasL, and sFasL in the death of photoreceptors following retinal detachment, we used two strains of mice that we previously identified as susceptible (BALB/c) and resistant (B6129SF2) to photoreceptor death following an induced retinal detachment. ${ }^{23}$ BALB/c FasL - /- mice were produced by an eight nucleotide deletion in the Fas $L$ gene that results in a splicing error and frameshift mutation (Supplementary Figure $1 \mathrm{~A}$ and B). B6129 $\triangle \mathrm{CS}$ mice were produced by an exchange mutation in FasL gene sequence, which replaces four residues bracketing two enzymatic cleavage sites (Supplementary Figure 1C). The retinas of FasL $-/-$ and $\triangle \mathrm{CS}$ mice were not significantly different from WT mice (Supplementary Figure 2). We first determined photoreceptor cell death over time after induction of retinal detachment by TdT-dUTP terminal nick-end labeling (TUNEL) assay in BALB/C WT and BALB/c FasL-/- mice. We previously demonstrated that the number of TUNEL-positive cells peaked on day $1 .{ }^{23}$ Therefore, we analyzed TUNEL-positive cell density in outer nuclear layer (ONL) at 0.5, 1, 3, 5, and 7 days after retinal detachment. The ONL/inner nuclear layer (INL) ratio was then assessed on day 7. A significant reduction in photoreceptor cell death was observed in BALB/c FasL - /- mice as compared with BALB/c WT mice at $24 \mathrm{~h}$ after retinal detachment (WT: $2359 \pm 202$ cells $/ \mathrm{mm}^{2}$ and FasL $-/-: 1105 \pm 154$ cells $/ \mathrm{mm}^{2} ;{ }^{\star \star} P<0.01$; Figure 1a). As expected, the other retinal layers showed no TUNELpositive cells at any time point (Figure 1b). Furthermore, BALB/c FasL - /- mice displayed significantly higher ONL/ INL ratios as compared with BALB/c WT mice 7 days after retinal detachment (WT: $1.30 \pm 0.06$ and FasL $-/-$ : $1.51 \pm 0.04 ;{ }^{\star} P<0.05$; Figure $\left.1 \mathrm{c}\right)$, suggesting that photoreceptor cell death was prevented in FasL-deficient mice.

$\triangle \mathrm{CS}$ mice have an exchange mutation in the FasL metalloproteinase cleavage site that prevents cleavage of $\mathrm{mFasL}$, resulting in increased levels of mFasL and no sFasL. ${ }^{22}$ Therefore, we speculated that even in the resistant B6129SF2 mouse strain, the increased levels of mFasL and the lack of sFasL in the B6129 $\triangle \mathrm{CS}$ mice would result in more photoreceptor death following retinal detachment as compared with B6129 WT mice. Consistent with our hypothesis, B6129 $\triangle \mathrm{CS}$ mice displayed significantly increased photoreceptor cell death at $24 \mathrm{~h}$ (WT: $654 \pm 128 \mathrm{cells} / \mathrm{mm}^{2}$ and $\Delta \mathrm{CS}$ : $1161 \pm 124$ cells $\left./ \mathrm{mm}^{2} ;{ }^{*} P<0.05\right)$, $120 \mathrm{~h}$ (WT: $134 \pm 33$ cells $/ \mathrm{mm}^{2}$ and $\Delta \mathrm{CS}: 286 \pm 30$ cells $\left./ \mathrm{mm}^{2} ;{ }^{\star} P<0.05\right)$, and $168 \mathrm{~h}$ (WT: $78 \pm 8 \mathrm{cells} / \mathrm{mm}^{2}$ and $\Delta \mathrm{CS}: 160 \pm 11 \mathrm{cells} / \mathrm{mm}^{2}$; $\left.{ }^{* * *} P<0.001\right)$ after retinal detachment (Figure 1d). The other retinal layers showed no TUNEL-positive cells at any time point (Figure 1e). Moreover, B6129 $\Delta \mathrm{CS}$ mice displayed significantly lower ONL/INL ratio as compared with B6129 WT mice (WT: $1.78 \pm 0.06$ and $\triangle \mathrm{CS}: 1.53 \pm 0.05 ;{ }^{\star} P<0.05$; Figure1f). We conclude from these results that preventing enzymatic cleavage of FasL significantly increased photoreceptor cell death following retinal detachment.

FasL mediates both photoreceptor apoptosis and necrosis after retinal detachment. To further investigate the type of photoreceptor cell death, we performed transmission electron microscopy (TEM) on the detached retina at $24 \mathrm{~h}$ after detachment (Figure 2a). BALB/c FasL-/- mice showed significantly less apoptotic photoreceptor cell death than BALB/C WT mice (WT: $21.0 \pm 1.8 \%$ and FasL-/-: $12.7 \pm 2.0 \%$; $\left.{ }^{\star} P<0.05\right)$. Interestingly, necrotic photoreceptor cell death was also significantly reduced in Fas $L-/-$ mice (WT: $8.1 \pm 1.1 \%$ and FasL $-/-$ : $4.6 \pm 0.3 \%$; ${ }^{\star} P<0.05$ ). These results indicate that abolishing FasL function in susceptible BALB/c mice can rescue both apoptotic and necrotic photoreceptor death following retinal detachment. On the other hand, in B6129SF2-resistant mice, $\triangle \mathrm{CS}$ mice 


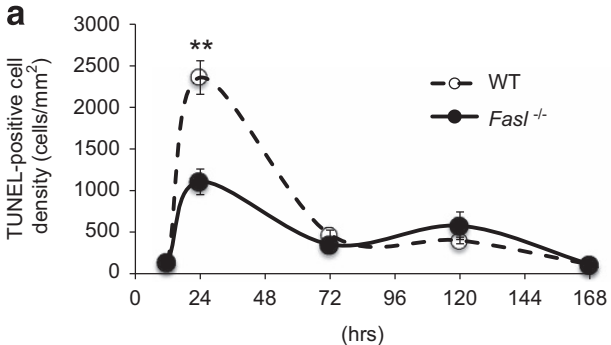

b
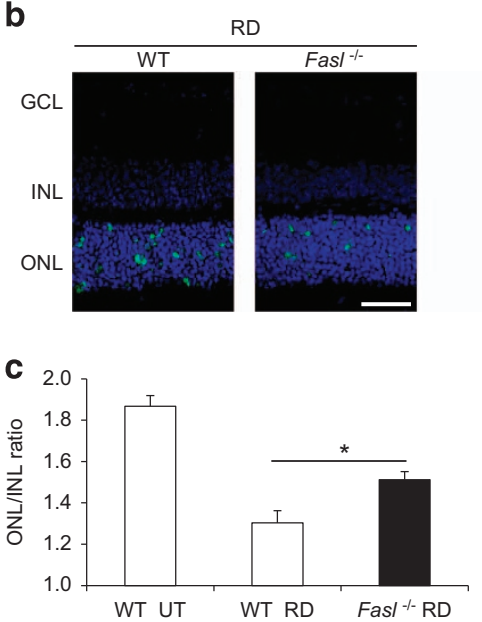

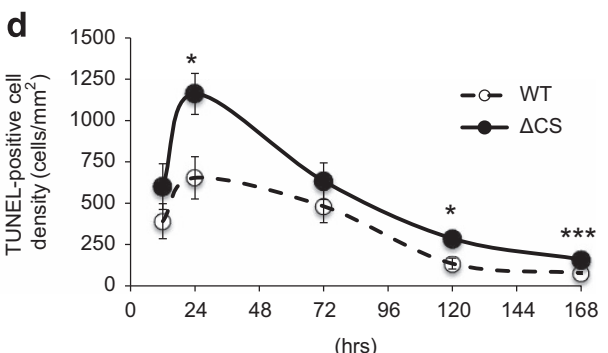

e
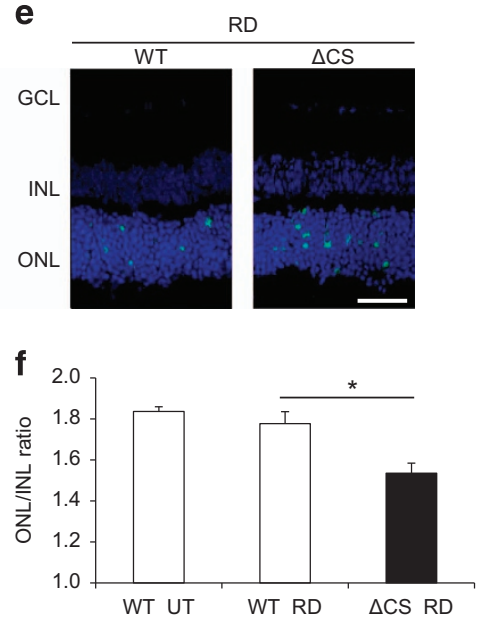

Figure 1 FasL mediates photoreceptor cell death induced by retinal detachment. (a) Time course of TUNEL-positive cell density in outer nuclear layer (ONL) in BALB/c WT and BALB/c FasL $-/-$ mice ( $n=6$ each group and time point). The peak of TUNEL-positive cell density was day 1 in both groups. BALB/c FasL $-/-$ mice showed significantly less photoreceptor cell death than BALB/c WT mice 1 day after retinal detachments $\left({ }^{\star *} P<0.01\right)$. (b) TUNEL (green) and TO-PRO-3 (blue) staining at $24 \mathrm{~h}$ after retinal detachment. Only ONL showed TUNEL-positive cells in both BALB/c WT and BALB/c FasL - /- mice. (c) ONL/INL (inner nuclear layer) ratio 7 days after retinal detachment ( $n=6$ each). ONL/NL ratio was significantly higher in BALB/c FasL $-/$ - than in BALB/c WT mice $\left({ }^{*} P<0.05\right)$. (d) Time course of TUNEL-positive cell density in ONL in B6129 WT and B6129 $\Delta$ CS mice ( $n=6$ each group and time point). The peak of TUNEL-positive cell density was on day 1 in both groups. B6129 $\Delta C S$ mice showed significantly more photoreceptor cell death than B6129 WT mice at $24 \mathrm{~h}\left({ }^{\star} P<0.05\right), 120 \mathrm{~h}\left({ }^{*} P<0.05\right)$, and $168 \mathrm{~h}\left({ }^{\star} P<0.001\right)$ after retinal detachment. (e) TUNEL (green) and TO-PRO-3 (blue) staining at $24 \mathrm{~h}$ after retinal detachment. Only ONL showed TUNEL-positive cells in both B6129 WTand B6129 $\Delta$ CS mice. (f) ONL/NL ratio 7 days after retinal detachment $(n=6$ each). ONL/NL ratio was significantly lower in B6129 $\Delta$ CS than in B6129 WT mice $\left({ }^{*} P<0.05\right)$. Scale bar, $50 \mu \mathrm{m}$. The graphs show mean $\pm S . E . M$. GCL, ganglion cell layer; UT, untreated

a
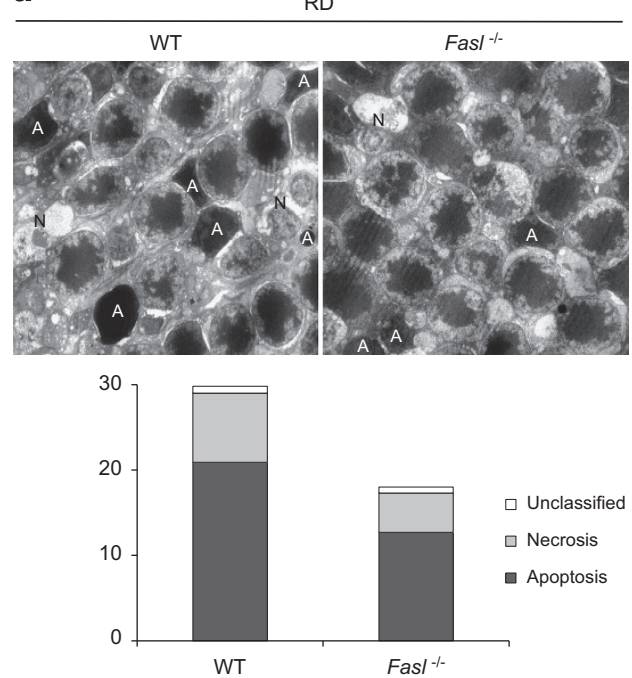

b
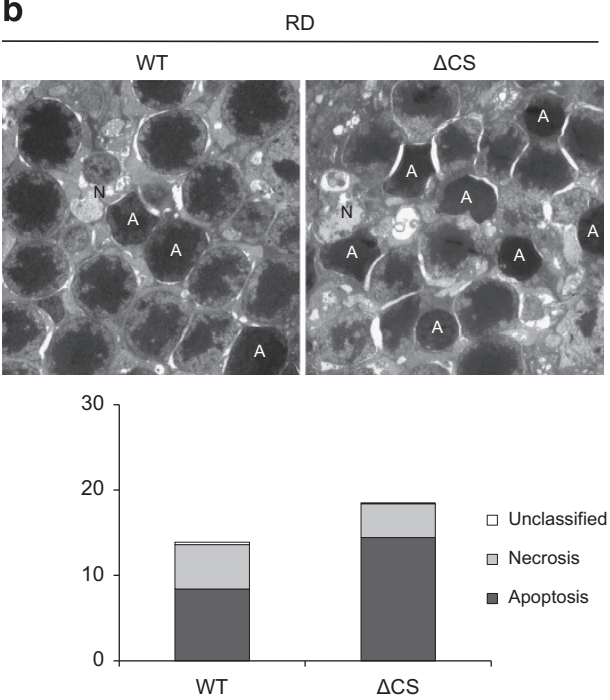

Figure 2 FasL mediates both photoreceptor apoptosis and necrosis after retinal detachment. (a) BALB/c FasL - / - mice showed significantly less apoptotic and necrotic photoreceptor cell deaths than BALB/c WT mice at $24 \mathrm{~h}$ after retinal detachment $(P<0.05$ each). (b) B6129 $\Delta$ CS mice exhibited significantly more apoptotic photoreceptor cell death than B6129 WT mice at $24 \mathrm{~h}$ after retinal detachment $(P<0.05$ each), whereas there was no significant difference in necrotic photoreceptor cell death between them. A, apoptosis; N, necrosis 
displayed significantly more apoptotic photoreceptor cell death than WT mice (WT: $8.4 \pm 0.9 \%$ and $\triangle \mathrm{CS}$ : $14.4 \pm 1.5 \%$; $\left.{ }^{\star} P<0.05\right)$. However, there was no significant difference in necrotic photoreceptor cell death between the two groups (WT: $5.2 \pm 0.9 \%$ and $\Delta \mathrm{CS}$ : $4.0 \pm 0.4 \%$ ). These results indicate that preventing enzymatic cleavage of FasL accelerated apoptotic (but not necrotic) photoreceptor cell death.

Retinal detachment-induced inflammatory cytokine expression is reduced in Fas $L-/-$ mice and increased in $\mathbf{S C S}$ mice. We previously demonstrated that monocyte chemoattractant protein 1 (MCP-1) is an essential mediator of early infiltration of macrophage/microglia after retinal detachment. ${ }^{24}$ Zacks et al. ${ }^{25,26}$ observed that interleukin 6 (IL-6) was also increased in the retina after retinal detachment using gene microarray analysis. IL- 6 is one of the stress-response genes related to inflammation as well as hematopoiesis, angiogenesis, cell differentiation, and neuronal survival, which can be produced by retinal cells other than macrophages. We evaluated MCP-1 and IL-6 expression levels by ELISA at $24 \mathrm{~h}$ after retinal detachment in the whole retina. In susceptible BALB/c mice, MCP-1 was significantly lower in FasL-/- as compared with WT mice (Figure 3a). However, there was no significant difference in IL-6 (Figure 3b). In contrast, in resistant B6129SF2 mice, $\triangle \mathrm{CS}$ mice showed a significant increase in both MCP-1 and IL-6 levels compared with WT mice (Figures $3 c$ and d). These data indicate FasL triggers an increase in pro-inflammatory MCP-1 and IL-6, which is further increased when enzymatic cleavage of FasL is blocked.

Inflammatory cell infiltration after retinal detachment is accelerated in Fas $L-/-$ mice. We next analyzed the time course of migrating $\mathrm{CD} 11 \mathrm{~b}+$ macrophage/microglial cells following retinal detachment. Interestingly, despite the lower MCP-1 levels observed in the absence of FasL, the infiltration of CD11b-positive cells was accelerated, resulting in a significant increase in the number of infiltrating CD11bpositive cells in FasL - /- compared with WT mice $24 \mathrm{~h}$ after retinal detachment (Supplementary Figure S3A and B). By $72 \mathrm{~h}$, the number of CD11b-positive cells was equal in both FasL $-/$ - and WT mice. On the other hand, although $\Delta \mathrm{CS}$ mice showed significantly higher MCP-1 and IL-6 levels compared with WT mice, the number of infiltrating CD11bpositive cells was not significantly different at all time points examined (Supplementary Figure S3C and D).

Differential M1 and M2 subtype of subretinal macrophages after induction of retinal detachment. Classically and alternatively activated macrophages (M1 and M2, respectively) have been reported to display opposing
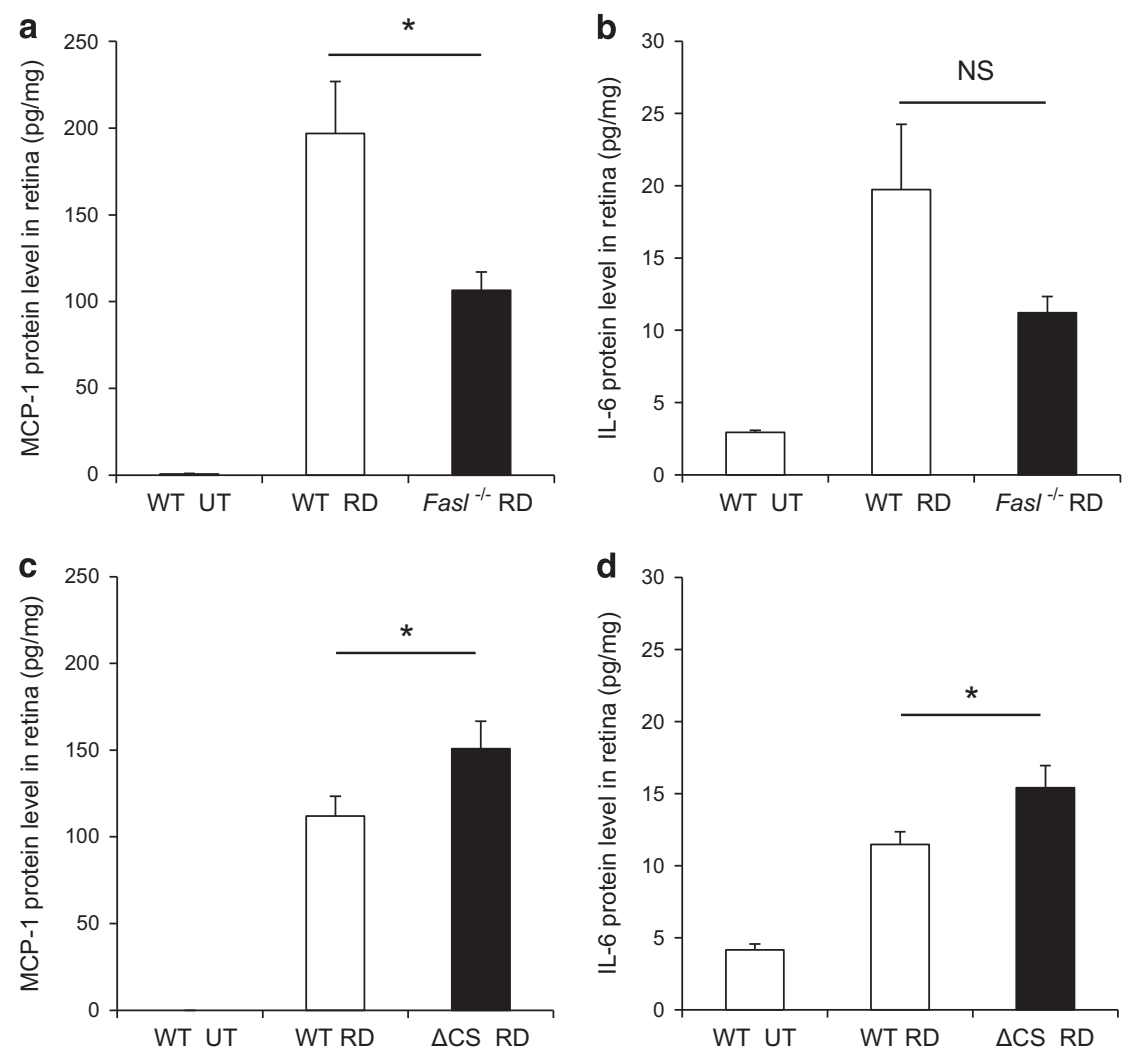

Figure 3 Retinal detachment-induced inflammatory cytokine expression is reduced in FasL - / - mice but increased in $\triangle C S$ mice. (a and b) ELISA to detect MCP-1 (a) and IL-6 (b) in the BALB/c WT and BALB/c FasL - / - retinas at $24 \mathrm{~h}$ after retinal detachment ( $n=8$ each). MCP-1 generation was significantly suppressed in BALB/c FasL $-/-$ mice $\left({ }^{*} P<0.05\right)$. IL-6 expression was reduced (but not significant) in BALB/c FasL $-/$ - than in BALB/c WT mice. (c and d) ELISA to detect MCP-1 (c) and IL-6 (d) in the B6129 WT and B6129 $\triangle$ CS retinas at $24 \mathrm{~h}$ after retinal detachment $(n=8$ each). MCP-1 and IL-6 generation was significantly increased in B6129 $\Delta$ CS than in B6129 WT mice $\left({ }^{*} P<0.05\right)$ 
functions during retinal degeneration. ${ }^{27,28}$ Ccr2 and Ly6c are considered as M1 markers, while Cx3cr1 is a M2 marker. ${ }^{27-29}$ To determine the macrophage subtypes within the subretinal space following detachment of the retina, we evaluated Ccr2, Ly6c, and Cx3cr1 mRNA expression in macrophage/microglia cells isolated on days 1 and 7 via laser capture microdissection (LCM) followed by quantitative real-time PCR (qPCR). In susceptible BALB/c mice, both WT and FasL-/_ mice displayed significantly higher levels of Ccr2 and $L y 6 c$ mRNA at day 1 as compared with day 7 (Figures $4 \mathrm{a}$ and b), while Cx3cr1 mRNA was significantly higher at day 7 as compared with day 1 (Figure 4c). However, there were no significant differences in the expression of Ccr2, Ly6c, and Cx3cr1 between WT and FasL - /- mice. Resistant B6129SF2 mice showed a similar pattern of expression, and there were no significant differences between $\triangle \mathrm{CS}$ and WT mice (Figures $4 \mathrm{~d}-\mathrm{f}$ ). Although the difference of some mRNA expressions between days 1 and 7 did not achieve statistical significance, there was an observable trend, and larger sample sizes might lead to statistical significance. These results indicate that, while the macrophages migrating into the subretinal space in this retinal detachment model are differentially activated depending on the time after detachment (with M1 seen in the early time points and M2 seen at later time points), the differences in subtypes were not affected by FasL.

Soluble Fas ligand rescued photoreceptor cells from death after retinal detachment. In contrast to the pro-death effects of mFasL, sFasL is reported to have pro-survival cell effects. ${ }^{17,18}$ We previously demonstrated that the intravitreal injection of recombinant sFasL prevented TNFa-triggered RGC death, ${ }^{22}$ suggesting that sFasL might antagonize the activity of mFasL. In the current study, we injected recombinant murine sFasL $(10 \mathrm{ng} / 1 \mu \mathrm{l})$ or control phosphate-buffered saline (PBS) into the subretinal space when creating retinal detachment in B6129 $\triangle \mathrm{CS}$ mice. The eyes treated with sFasL showed significantly reduced photoreceptor cell death as compared with control eyes (CTR: $1554 \pm 288$ cells $/ \mathrm{mm}^{2}$ and sFasL: $767 \pm 123$ cells $/ \mathrm{mm}^{2} ; P=0.045$; Figures $5 \mathrm{a}$ and b). This indicates that sFasL antagonizes the mFasL activity in detached retinas and prevents photoreceptor cell death.

\section{Discussion}

This study demonstrated that Fas/FasL-triggered cell death accounts for a significant amount of photoreceptor cell loss following retinal detachment, but not all cell death is due to
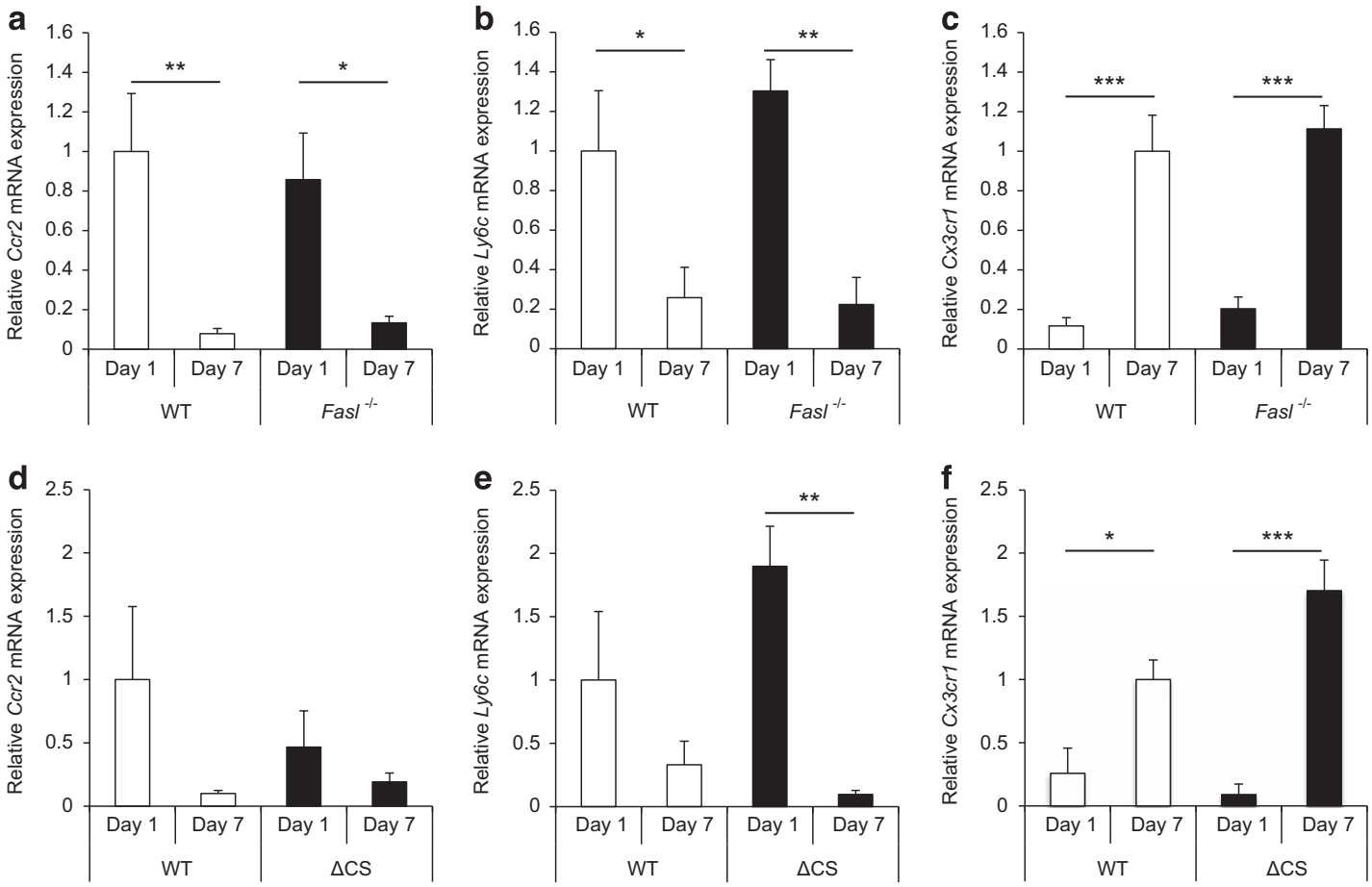

Figure 4 Classically and alternatively activated subretinal macrophages after induction of retinal detachment. (a-c) Ccr2, Ly6c, and Cx3cr1 mRNAs expression in the subretinal space was evaluated by LCM followed by qPCR using BALB/c WT and BALB/c FasL $-/$ - mice at 1 and 7 days after retinal detachment ( $n=6$ each group and time point). Ccr2 mRNA was significantly higher in day 1 than in day 7 in each group (WT: ${ }^{* *} P<0.01$, FasL $\left.-/-:{ }^{*} P<0.05\right)($ a). Ly6c mRNA was significantly higher in day 1 as compared with day 7 (WT: ${ }^{*} P<0.05$, FasL $-/-:{ }^{* *} P<0.01$ ) (b). On the other hand, Cx3cr1 mRNA was significantly higher in day 7 than in day 1 (WT: ${ }^{* * *} P<0.001, F a s L-/-$ : $\left.{ }^{* * \star} P<0.001\right)$ (c). There were no significant differences in the three mRNAs expression between BALB/c WTand BALB/c FasL $-/-$ mice. (d-f) $C c r 2, L y 6 c$, and $C \times 3 c r 1 \mathrm{mRNAs}$ expression in the subretinal space in B6129 WTand B6129 $\Delta$ CS mice at 1 and 7 days after retinal detachment ( $n=6$ each group and time point). Ccr2 mRNA tended to be higher in day 1 than in day 7 in each group; however, the differences did not achieve statistical significance (d). Ly6c mRNA was significantly higher in day 1 as compared with day 7 in B6129 $\Delta$ CS mice $\left.{ }^{* *} P<0.01\right)$. B6129 WT mice showed higher Ly6c mRNA in day 1 than in day 7 ; however, the difference did not reach statistical significance (e). Cx3cr1 mRNA was significantly higher in day 7 than in day 1 (WT: ${ }^{*} P<0.05, \Delta \mathrm{CS}:{ }^{* * *} P<0.001$ ) (f). There were no significant differences in the three mRNAs expression between B6129 WT and B6129 $\Delta$ CS mice. The graphs show mean \pm S.E.M. 
a

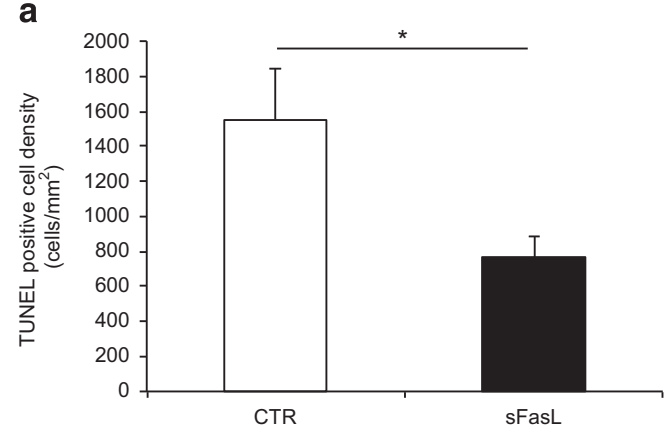

b

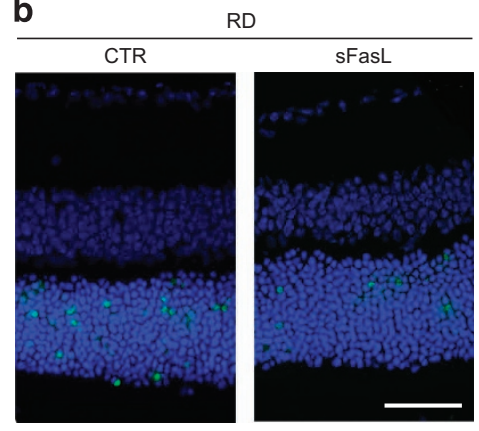

Figure 5 Subretinal injection of soluble Fas ligand attenuates photoreceptor cell death after retinal detachment. (a) TUNEL-positive cell density in the outer nuclear layer (ONL) in B6129 $\Delta$ CS mice at $24 \mathrm{~h}$ after retinal detachment. Recombinant murine soluble Fas ligand (sFasL) or control phosphate-buffered saline (CTR) was injected into the subretinal space when creating retinal detachment $(n=12$ each group). The eyes with sFasL showed significantly reduced photoreceptor cell death as compared with control eyes $\left({ }^{*} P<0.05\right)$. (b) TUNEL (green) and TO-PRO-3 (blue) staining at $24 \mathrm{~h}$ after retinal detachment. The graphs show mean \pm S.E.M. Scale bar, $50 \mu \mathrm{m}$

Fas/FasL, as the complete absence of FasL in KO mice did not completely abolish photoreceptor cell death. In addition, we demonstrated that the function of FasL was dependent upon the form of FasL that was expressed. Membrane-bound FasL triggered photoreceptor cell death, while soluble FasL exhibited neuroprotective effects, indicating that enzymemediated cleavage of FasL determines, in part, the extent of vision loss following retinal detachment. Moreover, it also indicates that treatment with sFasL could significantly reduce photoreceptor cell loss in patients with detached retinas. There have been several previous reports examining the function of Fas signaling in photoreceptor cell death after retinal detachment using Fas or FasL mutant mice., ${ }^{6,20}$ Hisatomi et al. $^{20}$ reported that neither Fas deficiency (Ipr/lpr) nor FasL deficiency (gld/gld) offered protection against photoreceptor cell death after retinal detachment. In contrast, Zacks et al. ${ }^{6}$ reported that $L P R$ mice (lacking a functional Fas receptor) showed significantly reduced photoreceptor cell death after retinal detachment as compared with control mice. They also demonstrated that detachment-induced photoreceptor cell death was rescued by subretinal injection of Fas-neutralizing antibody, small interfering RNA against the Fas-receptor transcript (siFAS), ${ }^{6}$ or a small peptide inhibitor (Met12). ${ }^{7}$ Moreover, Zacks et al. ${ }^{6}$ indicated that the LPR mice used by Hisatomi et al. ${ }^{20}$ were bred on a $\mathrm{C} 3 \mathrm{H}$ background, which carries the retinal degeneration 1 (rd1) allele., 60 Therefore, the use of these mice with an $r d 1$ mutation by Hisatomi et al. most likely confounded their results because the $r d 1$ retinal degeneration is driven predominantly by apoptosis-inducing factor and caspase $12 .{ }^{31}$ Moreover, the GLD and LPR mouse strains used by Zack et al. ${ }^{6}$ did not completely block Fas/FasL signaling since these mice have point mutations that allow residual activation of the Fas/FasL pathway, as reported by Karray et al. ${ }^{21}$ Unfortunately, FasLoverexpressing mice are not available, and we could not compare the photoreceptor cell death after retinal detachment between $\triangle \mathrm{CS}$ and FasL-overexpressing mice, which remains a limitation of our study.

Using B6129 mice that are resistant to retinal detachmentinduced photoreceptor cell death, we demonstrated that blocking enzymatic cleavage of FasL in B6129 $\Delta$ CS mice significantly increased the loss of photoreceptors following the retinal detachment. $\triangle \mathrm{CS}$ mice have an exchange mutation in the FasL metalloproteinase binding site that prevents cleavage of mFasL to produce sFasL. ${ }^{22}$ Conversely, in WT mice, metalloproteinases cleave mFasL to produce $\mathrm{sFasL}$, which reduces the Fas death signaling. ${ }^{13,17,22}$ This suggests that cleavage of FasL to produce $S F a s L$ is a protective mechanism to prevent photoreceptor cell death from Fas signaling after retinal detachment. Moreover, we previously reported using $\triangle \mathrm{CS}$ mice that $\mathrm{mFasL}$ triggers retinal ganglion cell death in two different models of glaucoma. ${ }^{22}$ Taken together, these results implicate FasL cleavage as a potential mechanism for limiting the neurotoxic activity of $\mathrm{mFasL}$ in the eye.

The modified retinal detachment model we used in this study, in which the height of the detachment was increased, consistently showed the peak of photoreceptor cell death on day $1,{ }^{32,33}$ whereas previous studies (including those from our own group) that used a more shallow detachment reported that the peak of photoreceptor death was on day $3 .^{20,34,35}$ These results are consistent with reports that photoreceptor cell death increases with the increasing height of retinal detachment. ${ }^{36,37}$ Photoreceptor cell damage in higher detachments may be more extensive compared with shallow detachments because of reduced diffusion of oxygen and essential nutrients from the choriocapillaris. ${ }^{38,39}$ Using our modified retinal detachment model, we create a detachment that is extremely bullous and persistent in $60 \%$ of the fundus, which appears to accelerate photoreceptor cell death in the detached retina more than other models.

In this study, we used two strains of mice that we previously demonstrated as susceptible (BALB/c) and resistant (B6129) to retinal detachment-induced photoreceptor death. When FasL was knocked out in susceptible BALB/c mice, there was a significant reduction in photoreceptor death. In contrast, when enzymatic cleavage was blocked in resistant B6129 $\Delta$ CS mice, there was a significant increase in photoreceptor death. Interestingly, TEM analysis revealed a reduction in both apoptotic and necrotic photoreceptor death in BALB/c FasL - /- mice. However, blocking the cleavage of FasL in B6129 $\triangle$ CS mice increased apoptotic photoreceptor death, but had no effect on necrotic cell death. These results suggest that both caspase-mediated apoptosis signaling and RIP-mediated necrotic signaling are attenuated in the 
absence of FasL. However, we speculate that increased mFasL in $\triangle \mathrm{CS}$ mice might preferentially trigger the apoptosis signaling pathway over the necrotic signaling pathway.

Macrophages infiltrate into the subretinal space after a retinal detachment. ${ }^{11,24,40-43}$ Hisatomi et al. ${ }^{40}$ reported that macrophages infiltrate from choroidal vessels into the subretinal space beyond the RPE layer. The RPE constitutively express FasL, which functions as an immune-privileged tissue 'barrier' that induces apoptosis of infiltrating Fas+ cells, thereby blocking cell infiltration. ${ }^{44,45}$ Moreover, it has also been reported that macrophages express Fas and readily undergo apoptosis when cultured in vitro with anti-Fas antibodies. $^{46,47}$ Using Ly6c, a maker expressed on bonemarrow derived monocytes, we recently demonstrated a significant increase in $L y 6 c \mathrm{mRNA}$ expression in the subretinal space on day 1 as compared with day 7 following retinal detachment. ${ }^{28}$ Taken together, these data predict that FasL expressed on RPE will inhibit macrophage infiltration into the subretinal space $24 \mathrm{~h}$ after retinal detachment. Our data are consistent with this prediction, since in the absence of FasL in BALB/c FasL - / - mice we observed a significant increase in the infiltration of CD11b-positive cells into the subretinal space following retinal detachment, even in the presence of reduced expression of the chemotactic factor MCP-1. Moreover, blocking the cleavage of FasL in B6129 $\triangle \mathrm{CS}$ mice, which increases the expression of pro-apoptotic $\mathrm{mFasL}$, resulted in a significant reduction in the infiltration of CD11b-positive cells into the subretinal space, even in the presence of elevated expression of MCP-1. Together, these data support the hypothesis that the pro-apoptotic mFasL expressed on the RPE inhibits macrophage infiltration into the subretinal space following retinal detachment. However, it is possible that CD11b-positive cells that migrated into the subretinal space might not be macrophages, but rather other immune cells (e.g., natural killer cells). FasL can affect the function of CD11b-positive cells and cause the discrepancy between MCP-1 expression and migration of CD11b-positive cells after retinal detachment, which remains to be elucidated.

It has been shown that classically activated (M1) and alternatively activated (M2) macrophages have opposite roles in inflammation and wound healing. ${ }^{48-50} \mathrm{M} 1$ macrophages generate high levels of pro-inflammatory cytokines and produce reactive nitrogen and oxygen intermediates. In contrast, M2 macrophages have immunoregulatory functions, promote tissue remodeling, express high levels of scavenger receptors, and display efficient phagocytic activity. In the current study, we demonstrated that the subtype of infiltrating macrophages changes with time after retinal detachment: M1 macrophages are the predominant cell type during the acute phase (day 1) and M2 macrophages are the predominant cell type during the late phase (day 7). In addition, the CD11bpositive cell density was higher in the late phase as compared with the acute phase, which corresponded with a higher frequency of TUNEL-positive cells in the acute phase. The switch from M1 to M2 subtype may be a partial reason for the endogenous neuroprotection seen after retinal detachment that leads to the observed decline in the rate of cell death over time. However, neither the mouse strain nor FasL had any effect on the timing or subtype of infiltrating macrophages during retinal detachment.
Murine sFasL is reported to be non-apoptotic and antiinflammatory, and can even act as an antagonist by blocking mFasL engagement with the Fas receptor. ${ }^{17,18}$ We previously demonstrated the neuroprotective effects of sFasL in a TNFa-triggered model of retinal ganglion cell neurotoxicity. ${ }^{22}$ In the current study, we simultaneously injected recombinant sFasL into the subretinal space when the retinal detachment was created, which attenuated the subsequent photoreceptor cell death. These data suggest that sFasL might antagonize the activity of mFasL and prevent photoreceptor cell death following retinal detachment.

In conclusion, using Fas $L-/-$ and $\triangle \mathrm{CS}$ mice, we demonstrated that the different forms of FasL display opposite functions following the retinal detachment: $\mathrm{mFasL}$ increased loss of photoreceptors, whereas sFasL inhibited cell death. In addition, $\mathrm{mFas} L$ expressed on the RPE may limit inflammatory M1 cell infiltration during the acute phase following retinal detachment, independent of the presence of pro-inflammatory cytokines and chemokines. This blockade was reduced during the later phase of retinal detachment allowing increased numbers of M2 macrophages into the wounded retina. Understanding the mechanisms of FasL-induced photoreceptor cell death and endogenous neuroprotection will provide new therapeutic targets for preventing loss of photoreceptors in retinal diseases affected by separation of photoreceptors from RPE, such as rhegmatogenous retinal detachment.

\section{Materials and Methods}

Animals. All animal experiments followed the guidelines of the ARVO Statement for the Use of Animals in Ophthalmic and Vision Research and were approved by the Animal Care Committee of the Massachusetts Eye and Ear Infirmary. FasL - / - mice on the BALB/c background were provided by Ann MarshakRothstein (Supplementary Figure S1). ${ }^{22}$ BALB/c mice were purchased from Charles River Laboratories (Wilmington, MA, USA) as control WT mice for FasL - /- mice. $\Delta$ CS mice on the B6129 background were provided by Ann Marshak-Rothstein (Supplementary Figure S1). ${ }^{22}$ B6129SF2 mice were purchased from Jackson Laboratories (Bar Harbor, ME, USA) as control WT mice for $\triangle \mathrm{CS}$ mice. Mice were fed standard laboratory chow and allowed free access to water in an air-conditioned room with a 12-h light/12-h dark cycle. All mice were used at postnatal $8 \pm 1$ weeks.

Creation of retinal detachment. We modified a previously reported method for creating experimental retinal detachments and achieved bullous and persistent retinal detachments. ${ }^{51}$ Briefly, mice were anesthetized with an intraperitoneal injection of a mixture of $60 \mathrm{mg} / \mathrm{kg}$ ketamine and $6 \mathrm{mg} / \mathrm{kg}$ xylazine, and pupils were dilated with topical phenylephrine $(5 \%)$ and tropicamide $(0.5 \%)$. The temporal conjunctiva at the posterior limbus was incised and detached from the sclera. A 30-gauge needle (BD, Franklin Lakes, NJ, USA) was used with the bevel pointed up to create a sclerotomy $1 \mathrm{~mm}$ posterior to the limbus. A scleral tunnel was created followed by scleral penetration into the choroid, which makes a self-sealing scleral wound. A corneal puncture was made with a 30-gauge needle to lower intraocular pressure. Then, a 34-gauge needle connected to a 10- $\mu$ l syringe (NanoFil $10 \mu \mathrm{l}$ syringe; WPI, Sarasota, FL, USA) with the bevel pointed down was inserted into the subretinal space and $4 \mu \mathrm{l}$ of $1 \%$ sodium hyaluronate (Provisc; Alcon, Fort Worth, TX, USA) was injected gently to detach the neurosensory retina from the underlying RPE. Approximately $60 \%$ of the temporal-nasal neurosensory retina was detached. For evaluation of the efficacy of sFasL to prevent photoreceptor cell death after retinal detachment, recombinant murine sFasL ( $10 \mathrm{ng} / 1 \mu$ l; R\&D Systems, Inc., Minneapolis, MN, USA) or control PBS was injected into the subretinal space followed by injection of $4 \mu \mathrm{l}$ of $1 \%$ sodium hyaluronate. Finally, cyanoacrylate surgical glue (WebglueTM; Patterson Veterinary, Devens, MA, USA) was put on the scleral wound, and the conjunctiva was reattached to the original position. Any eyes with subretinal hemorrhage were excluded from the study. 
TdT-dUTP terminal nick-end labeling. Following retinal detachments, eyes were enucleated at multiple time points and embedded in OCT compound (Tissue Tek; Sakura Finetec, Torrance, CA, USA). Serial sections of the eyes in the sagittal plane were cut at $10 \mu \mathrm{m}$ thickness on a cryostat (CM1850; Leica, Heidelberg, Nussloch, Germany) at $-20^{\circ} \mathrm{C}$ and prepared for staining. TUNEL assay was performed according to the manufacturer's protocol (ApoTag Fluorescein In Situ Apoptosis Detection Kit; Millipore, Billerica, MA, USA). Finally, sections were counterstained with TO-PRO-3. The number of TUNEL-positive cells was counted in the ONL, which contains the photoreceptors. The area of ONL was also measured by the Image $\mathrm{J}$ software (developed by Wayne Rasband, National Institutes of Health, Bethesda, MD, USA; available at http://rsb.info.nih.gov/ij/index.html), and then TUNELpositive cell density in ONL was calculated. A preliminary experiment revealed that the center of the retinal detachment had less variability of TUNEL-positive cell density (data not shown). Thus, TUNEL-positive cell density was evaluated using sections around $1000 \mu \mathrm{m}$ from the injection site. ${ }^{23,32}$ Photographs were taken by confocal microscopy using a HCX APOL 40x lens (Leica, Allendale, NJ, USA).

Evaluation of ONL/INL ratio. The ONL and INL areas of the detached retina were measured by the Image $\mathrm{J}$ software, and the ONL/NL ratio was calculated. Areas of abnormal retinal morphology were excluded so that uniform unbiased measurements can be obtained.

Transmission electron microscopy. TEM was performed as previously described. ${ }^{52}$ More than 200 photoreceptors per eye were photographed and subjected to quantification of cell death modes in a masked manner. Photoreceptors showing cellular shrinkage and nuclear condensation were defined as apoptotic cells, whereas photoreceptors associated with cellular and organelle swelling and discontinuities in plasma and nuclear membrane were defined as necrotic cells. Electron-dense granular materials were labeled simply as end-stage cell death/ unclassified, because these materials are reported to occur subsequently to both apoptotic and necrotic cell death.

ELISA for MCP-1 and IL-6. The levels of MCP-1 and IL- 6 were determined with mouse MCP-1 and IL-6 ELISA kits (R\&D Systems, Inc.), according to the manufacturer's protocol.

Immunohistochemistry. Sections were fixed in acetone for $5 \mathrm{~min}$, blocked in $2 \%$ skim milk for $20 \mathrm{~min}$, and incubated with rat anti-CD11b antibody (1:50; BD Biosciences, San Jose, CA, USA) at $4^{\circ} \mathrm{C}$ overnight. Alexa Fluor 488-conjugated goat anti-rat lgG was used as a secondary antibody and incubated at room temperature for $30 \mathrm{~min}$. Finally, sections were counterstained with TO-PRO-3.

Laser capture microdissection. LCM was performed with Leica LMD 7000 (Leica Microsystems, Buffalo Grove, IL, USA). After enucleation, eyes were frozen in OCT compound and cut into $40 \mu \mathrm{m}$ sections on RNase-free polyethylene naphthalate membrane slides (Leica Microsystems). The sections were fixed and dehydrated with 75,95 , and $100 \%$ ethanol. The tissues from subretinal space were microdissected and collected into $0.5-\mathrm{ml}$ tubes containing RNAlater (Life Technologies, Grand Island, NY, USA).

Measurement of mRNA expression by qPCR. The subretinal tissue dissected by LCM was used for qPCR. Total RNA was harvested using the RNeasy Plus Micro Kit (Qiagen, Valencia, CA, USA). cDNA was generated with Oligo-dT primer (Invitrogen, Camarillo, CA, USA) and Superscript III (Invitrogen) according to the manufacturer's instructions. Quantitative PCR was carried out using Ccr2 (Mm99999051_gH), Ly6c (Mm03009946_m1), Cx3cr1 (Mm02620111_s1), II6 (Mm00446190_m1), and Rn18s (Mm03928990_g1) TaqMan gene expression assay (Applied Biosystems, Foster City, CA, USA).

Statistical analysis. The results are expressed as the mean \pm S.E. Statistical differences between two groups were analyzed by Mann-Whitney $U$ test. Multiplegroup comparison was performed by two-way ANOVA followed by Bonferroni's posttest. The significance level was set at $P<0.05$ ( ${ }^{*}$ in figures), $P<0.01$ (** in figures), $P<0.001$ ( ${ }^{* *}$ in figures). Statistical analysis and graphing were performed using Prism Ver.5 (GraphPad Software, La Jolla, CA, USA).

\section{Conflict of Interest}

The authors declare no conflict of interest.
Acknowledgements. We thank Wendy Chao for her support in critical review. This work was supported by Foundation Lions Eye Research Fund (DGV); The Yeatts Family Foundation (DGV and JWM); The Loefffler Family Foundation (DGV and JWM); 2013 Macula Society Research Grant award (DGV); Bausch \& Lomb Vitreoretinal Fellowship (HM); Grant-in-Aid for Young Scientists (B) from Japan Society for the Promotion of Science (HM); Grant-in-Aid from Novartis Pharma K.K. Tokyo, Japan (HM); a RPB Physician Scientist Award (DGV) and unrestricted grant (JWM) from the Research to Prevent Blindness Foundation; NEI R21EY023079-01/ A1 (DGV) and NEI grant EY014104 (MEEI Core Grant). The content is solely the responsibility of the authors and does not necessarily represent the official views of the National Eye Institute or the National Institutes of Health.

1. Mitry D, Fleck BW, Wright AF, Campbell H, Charteris DG. Pathogenesis of rhegmatogenous retinal detachment: predisposing anatomy and cell biology. Retina 2010; 30: 1561-1572.

2. Dunaief JL, Dentchev T, Ying GS, Milam AH. The role of apoptosis in age-related macular degeneration. Arch Ophthalmol 2002; 120: 1435-1442.

3. Barber AJ, Lieth E, Khin SA, Antonetti DA, Buchanan AG, Gardner TW. Neural apoptosis in the retina during experimental and human diabetes. Early onset and effect of insulin. J Clin Invest 1998; 102: 783-791.

4. Hellstrom A, Smith LE, Dammann O. Retinopathy of prematurity. Lancet 2013; 382: 1445-1457.

5. Zacks DN, Zheng QD, Han Y, Bakhru R, Miller JW. FAS-mediated apoptosis and its relation to intrinsic pathway activation in an experimental model of retinal detachment. Invest Ophthalmol Vis Sci 2004; 45: 4563-4569.

6. Zacks DN, Boehlke C, Richards AL, Zheng QD. Role of the Fas-signaling pathway in photoreceptor neuroprotection. Arch Ophthalmol 2007; 125: 1389-1395.

7. Besirli CG, Chinskey ND, Zheng QD, Zacks DN. Inhibition of retinal detachment-induced apoptosis in photoreceptors by a small peptide inhibitor of the fas receptor. Invest Ophthalmol Vis Sci 2010; 51: 2177-2184.

8. Holler N, Zaru R, Micheau O, Thome M, Attinger A, Valitutti S et al. Fas triggers an alternative, caspase-8-independent cell death pathway using the kinase RIP as effector molecule. Nat Immunol 2000; 1: 489-495.

9. Degterev A, Huang Z, Boyce M, Li Y, Jagtap P, Mizushima N et al. Chemical inhibitor of nonapoptotic cell death with therapeutic potential for ischemic brain injury. Nat Chem Biol 2005; 1: 112-119.

10. He S, Wang L, Miao L, Wang T, Du F, Zhao L et al. Receptor interacting protein kinase-3 determines cellular necrotic response to TNF-alpha. Cell 2009; 137: 1100-1111.

11. Trichonas G, Murakami $Y$, Thanos A, Morizane $Y$, Kayama M, Debouck CM et al. Receptor interacting protein kinases mediate retinal detachment-induced photoreceptor necrosis and compensate for inhibition of apoptosis. Proc Natl Acad Sci USA 2010; 107: 21695-21700.

12. Suda T, Takahashi T, Golstein P, Nagata S. Molecular cloning and expression of the Fas ligand, a novel member of the tumor necrosis factor family. Cell 1993; 75: 1169-1178.

13. Tanaka M, Suda T, Takahashi T, Nagata S. Expression of the functional soluble form of human fas ligand in activated lymphocytes. EMBO J 1995; 14: 1129-1135.

14. Varadhachary AS, Edidin M, Hanlon AM, Peter ME, Krammer PH, Salgame $P$. Phosphatidylinositol 3'-kinase blocks CD95 aggregation and caspase-8 cleavage at the death-inducing signaling complex by modulating lateral diffusion of CD95. J Immunol 2001; 166: 6564-6569.

15. Huang DC, Hahne M, Schroeter M, Frei K, Fontana A, Villunger A et al. Activation of Fas by FasL induces apoptosis by a mechanism that cannot be blocked by $\mathrm{Bcl}-2$ or $\mathrm{Bcl}-\mathrm{x}(\mathrm{L})$. Proc Natl Acad Sci USA 1999; 96: 14871-14876.

16. Jang S, Krammer PH, Salgame P. Lack of proapoptotic activity of soluble CD95 ligand is due to its failure to induce CD95 oligomers. J Interferon Cytokine Res 2003; 23: 441-447.

17. Hohlbaum AM, Moe S, Marshak-Rothstein A. Opposing effects of transmembrane and soluble Fas ligand expression on inflammation and tumor cell survival. J Exp Med 2000; 191: 1209-1220.

18. Suda T, Hashimoto H, Tanaka M, Ochi T, Nagata S. Membrane Fas ligand kills human peripheral blood T lymphocytes, and soluble Fas ligand blocks the killing. J Exp Med 1997; 186: 2045-2050.

19. Griffith TS, Brunner T, Fletcher SM, Green DR, Ferguson TA. Fas ligand-induced apoptosis as a mechanism of immune privilege. Science 1995; 270: 1189-1192.

20. Hisatomi T, Sakamoto T, Murata T, Yamanaka I, Oshima Y, Hata Y et al. Relocalization of apoptosis-inducing factor in photoreceptor apoptosis induced by retinal detachment in vivo. Am J Pathol 2001; 158: 1271-1278.

21. Karray S, Kress C, Cuvellier S, Hue-Beauvais C, Damotte D, Babinet C et al. Complete loss of Fas ligand gene causes massive lymphoproliferation and early death, indicating a residual activity of gld allele. J Immunol 2004; 172: 2118-2125.

22. Gregory MS, Hackett CG, Abernathy EF, Lee KS, Saff RR, Hohlbaum AM et al. Opposing roles for membrane bound and soluble Fas ligand in glaucoma-associated retinal ganglion cell death. PLoS One 2011; 6: e17659.

23. Matsumoto H, Kataoka K, Tsoka P, Connor KM, Miller JW, Vavvas DG. Strain difference in photoreceptor cell death after retinal detachment in mice. Invest Ophthalmol Vis Sci 2014; 55: $4165-4174$. 
24. Nakazawa T, Hisatomi T, Nakazawa C, Noda K, Maruyama K, She H et al. Monocyte chemoattractant protein 1 mediates retinal detachment-induced photoreceptor apoptosis. Proc Natl Acad Sci USA 2007; 104: 2425-2430.

25. Zacks DN, Han Y, Zeng Y, Swaroop A. Activation of signaling pathways and stress-response genes in an experimental model of retinal detachment. Invest Ophthalmol Vis Sci 2006; 47 1691-1695.

26. Zacks DN. Gene transcription profile of the detached retina (An AOS Thesis). Trans Am Ophthalmol Soc 2009; 107: 343-382.

27. London A, Itskovich E, Benhar I, Kalchenko V, Mack M, Jung S et al. Neuroprotection and progenitor cell renewal in the injured adult murine retina requires healing monocyte-derived macrophages. J Exp Med 2011; 208: 23-39.

28. Fukuda S, Nagano M, Yamashita T, Kimura K, Tsuboi I, Salazar G et al. Functional endothelial progenitor cells selectively recruit neurovascular protective monocyte-derived $\mathrm{F} 4 / 80(+) / \mathrm{Ly} 6 \mathrm{c}(+)$ macrophages in a mouse model of retinal degeneration. Stem Cells 2013; 31: $2149-2161$.

29. Novak ML, Koh TJ. Phenotypic transitions of macrophages orchestrate tissue repair Am J Pathol 2013; 183: 1352-1363.

30. Chang B, Hawes NL, Hurd RE, Davisson MT, Nusinowitz S, Heckenlively JR. Retinal degeneration mutants in the mouse. Vision Res 2002; 42: 517-525

31. Sanges D, Comitato A, Tammaro R, Marigo V. Apoptosis in retinal degeneration involves cross-talk between apoptosis-inducing factor (AIF) and caspase-12 and is blocked by calpain inhibitors. Proc Natl Acad Sci USA 2006; 103: 17366-17371.

32. Matsumoto $\mathrm{H}$, Murakami $\mathrm{Y}$, Kataoka K, Lin H, Connor KM, Miller JW et al. Mammalian STE20-like kinase 2, not kinase 1, mediates photoreceptor cell death during retinal detachment. Cell Death Dis 2014; 5: e1269.

33. Matsumoto H, Kataoka K, Tsoka PA, Connor KM, Miller JW, Vavvas DG. Strain difference in photoreceptor cell death after retinal detachment in mice. Invest Ophthalmol Vis Sci 2014; 55: 4165-4174.

34. Hisatomi T, Sakamoto T, Goto Y, Yamanaka I, Oshima Y, Hata Y et al. Critical role of photoreceptor apoptosis in functional damage after retinal detachment. Curr Eye Res 2002 24: 161-172.

35. Yang L, Bula D, Arroyo JG, Chen DF. Preventing retinal detachment-associated photoreceptor cell loss in Bax-deficient mice. Invest Ophthalmol Vis Sci 2004; 45: 648-654.

36. Machemer R. Experimental retinal detachment in the owl monkey. IV. The reattached retina. Am J Ophthalmol 1968; 66: 1075-1091.

37. Ross W, Lavina A, Russell M, Maberley D. The correlation between height of macula detachment and visual outcome in macula-off retinal detachments of $<$ or $=7$ days duration. Ophthalmology 2005; 112: 1213-1217.

38. Mervin K, Valter K, Maslim J, Lewis G, Fisher S, Stone J. Limiting photoreceptor death and deconstruction during experimental retinal detachment: the value of oxygen supplementation. Am J Ophthalmol 1999; 128: 155-164.

39. Lewis GP, Talaga KC, Linberg KA, Avery RL, Fisher SK. The efficacy of delayed oxygen therapy in the treatment of experimental retinal detachment. Am J Ophthalmol 2004; 137: 1085-1095.

40. Hisatomi T, Sakamoto T, Sonoda KH, Tsutsumi C, Qiao H, Enaida $\mathrm{H}$ et al. Clearance of apoptotic photoreceptors: elimination of apoptotic debris into the subretinal space and macrophage-mediated phagocytosis via phosphatidylserine receptor and integrin alphavbeta3. Am J Pathol 2003; 162: 1869-1879.

41. Nakazawa T, Kayama M, Ryu M, Kunikata H, Watanabe R, Yasuda M et al. Tumor necrosis factor-alpha mediates photoreceptor death in a rodent model of retinal detachment. Invest Ophthalmol Vis Sci 2011; 52: 1384-1391.

42. Roh MI, Murakami Y, Thanos A, Vavvas DG, Miller JW. Edaravone, an ROS scavenger, ameliorates photoreceptor cell death after experimental retinal detachment. Invest Ophthalmol Vis Sci 2011; 52: 3825-3831.

43. Mantopoulos D, Murakami Y, Comander J, Thanos A, Roh M, Miller JW et al. Tauroursodeoxycholic acid (TUDCA) protects photoreceptors from cell death after experimental retinal detachment. PLoS One 2011; 6: e24245.

44. Kaplan HJ, Leibole MA, Tezel T, Ferguson TA. Fas ligand (CD95 ligand) controls angiogenesis beneath the retina. Nat Med 1999; 5: 292-297.

45. Wenkel $\mathrm{H}$, Streilein JW. Evidence that retinal pigment epithelium functions as an immuneprivileged tissue. Invest Ophthalmol Vis Sci 2000; 41: 3467-3473.

46. Richardson BC, Lalwani ND, Johnson KJ, Marks RM. Fas ligation triggers apoptosis in macrophages but not endothelial cells. Eur J Immunol 1994; 24: 2640-2645.

47. Um HD, Orenstein JM, Wahl SM. Fas mediates apoptosis in human monocytes by a reactive oxygen intermediate dependent pathway. J Immunol 1996; 156: 3469-3477.

48. Mantovani A, Sozzani S, Locati M, Allavena P, Sica A. Macrophage polarization: tumorassociated macrophages as a paradigm for polarized M2 mononuclear phagocytes. Trends Immunol 2002; 23: 549-555.

49. Gordon S, Martinez FO. Alternative activation of macrophages: mechanism and functions. Immunity 2010; 32: 593-604.

50. Sica A, Mantovani A. Macrophage plasticity and polarization: in vivo veritas. J Clin Invest 2012; 122: 787-795.

51. Matsumoto H, Miller JW, Vavvas DG. Retinal detachment model in rodents by subretinal injection of sodium hyaluronate. J Vis Exp 2013; e-pub ahead of print 11 September 2013; doi:10.3791/50660.

52. Hisatomi T, Nakazawa T, Noda K, Almulki L, Miyahara S, Nakao S et al. HIV protease inhibitors provide neuroprotection through inhibition of mitochondrial apoptosis in mice. J Clin Invest 2008; 118: 2025-2038.

(i) Cell Death and Disease is an open-access journal published by Nature Publishing Group. This work is licensed under a Creative Commons Attribution 4.0 International License. The images or other third party material in this article are included in the article's Creative Commons license, unless indicated otherwise in the credit line; if the material is not included under the Creative Commons license, users will need to obtain permission from the license holder to reproduce the material. To view a copy of this license, visit http://creativecommons.org/licenses/by/4.0/ 